\title{
Epidemiología de neutropenia febril en pacientes adultos con leucemia aguda y linfoma. Estudio de cohorte en hospitales público y privado de Santiago, Chile
}

\author{
Ricardo Rabagliati, Pablo Bertín, Inés Cerón, Hernán Rojas, Isabel Domínguez, Álvaro Vera, \\ Leonardo Siri, Jimena Flores, Paulina Fernández, Marco Pérez y Rolando De La Cruz
}

Epidemiology of febrile neutropenia in adult patients with acute leukemia and lymphoma. Cohort study of public and private hospital of Santiago, Chile

Introduction: Febrile neutropenia (FN) is a common complication of patients undergoing chemotherapy (QMT). Clinical presentation is varied, from mild fever to severe sepsis with invasive bacterial infection (IBI) or invasive fungal infection (IFI), with great impact on prognosis and patient mortality. Patients and Methods: Prospective cohort study of FN episodes in adult patients with acute leukemia (AL) or lymphoma (L), diagnosed and treated at the Hospital Clínico Universidad Católica and Hospital Dr. Sótero del Río in Santiago from April 2010 to January 2012. Results: 130 patients were included with 105 episodes of NF, with an incidence of 0.65 per 100 days of observation, higher in AL than L (1.31 vs $0.25, \mathrm{p}=0.001)$. Etiology or clinical focus was documented in $67(63.8 \%)$ episodes, with IBI in $33(31.4 \%)$ and IFI in $21(20 \%)$ cases. Mortality related to infection occurred in $4(6.2 \%)$ patients. Conclusions: This study reports that the FN incidence and frequency of IBI and IFI during episodes are higher in AL vs. L. It is necessary to evaluate the impact of interventions to reduce its incidence, including the benefit and risk of using antibacterial and antifungal prophylaxis in high-risk subgroups.

Key words: Febrile neutropenia, leukemia, lymphoma, invasive bacterial infections, invasive fungal disease.

Palabras clave: Neutropenia febril, leucemia, linfoma, infección bacteriana invasora, enfermedad fúngica invasora.

\section{Introducción}

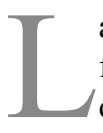

a neutropenia febril (NF) es una complicación frecuente en pacientes con cáncer, sometidos a quimioterapia (QMT) citotóxica ${ }^{1}$. Su presentación clínica es amplia, desde fiebre de intensidad variable, sin otros síntomas acompañantes, a sepsis grave con infección bacteriana invasora (IBI) o infección fúngica invasora $(\mathrm{IFI})^{2}$. Como consecuencia de la NF se generan aumentos de los costos globales de atención, retrasos en el inicio de ciclos posteriores de QMT con potencial impacto en el pronóstico oncológico, y mortalidad que alcanza hasta $8 \%$ por episodio ${ }^{3}$.

La frecuencia de NF depende de varios factores, entre ellos el tipo de cáncer, siendo más frecuente en pacientes con cánceres hematológicos ${ }^{4}$. Según la literatura médica internacional es esperable que se presente NF en más de 80\% de los ciclos de QMT de pacientes hematológicos, mientras que en los pacientes con cánceres de órganos sólidos es entre 10 y $50 \%$ de los ciclos ${ }^{4}$. Adicionalmente se ha descrito mayor gravedad y mayor frecuencia de IBI e IFI en los episodios de NF en pacientes con cáncer hematológico que en los pacientes con cánceres de órganos sólidos ${ }^{5}$. Durante los últimos años, tanto en niños como adultos con cáncer, la investigación de la epidemiología de la NF ha permitido reconocer grupos con diferente riesgo de complicaciones, quienes pueden ser identificados precozmente a través de modelos de predicción, planteándose diferentes estrategias terapéuticas, desde su manejo ambulatorio para los con menor riesgo a enfrentamiento más enérgico para aquellos con mayor riesgo ${ }^{6-11}$. Existen pocos datos publicados sobre la epidemiología de NF en pacientes adultos con cáncer en nuestro país, tanto en el ámbito público ${ }^{12,13}$ como privado $^{5}$. A la fecha, no se han publicado datos de incidencia, factores determinantes de riesgo y mortalidad de NF en grupos específicos de paciente por patología oncológica, que incluya simultáneamente centros públicos y privados.

Es necesario conocer la incidencia de NF en leucemia aguda (LA) y linfoma (L), sus factores de riesgo y la frecuencia de IBI/IFI en adultos chilenos que permita constituir una base para evaluar el impacto de futuras medidas de intervención. El objetivo principal de este estudio fue:

- Determinar la incidencia de NF en pacientes con LA o L en tratamiento de QMT.
Facultad de Medicina, Pontificia Universidad Católica de Chile, Santiago.

Dpto. de Enfermedades Infecciosas del Adulto (RR, LS, JF). Dpto. de Hematología y Oncología (PB) Gerencia de Tecnología en información (MP).

Departamento de Salud Pública (R de la C). Hospital Dr. Sótero del Río. Santiago, Chile.

Unidad de Infectología (IC, ID, AV). Unidad de Hematología (HR, PF).

Financiamiento: Proyecto FONIS SA091211.

Recibido: 29 de marzo de 2014 Aceptado: 12 de junio de 2014

Correspondencia a: Ricardo Rabagliati Rabagliati rabagli@med.puc.cl 


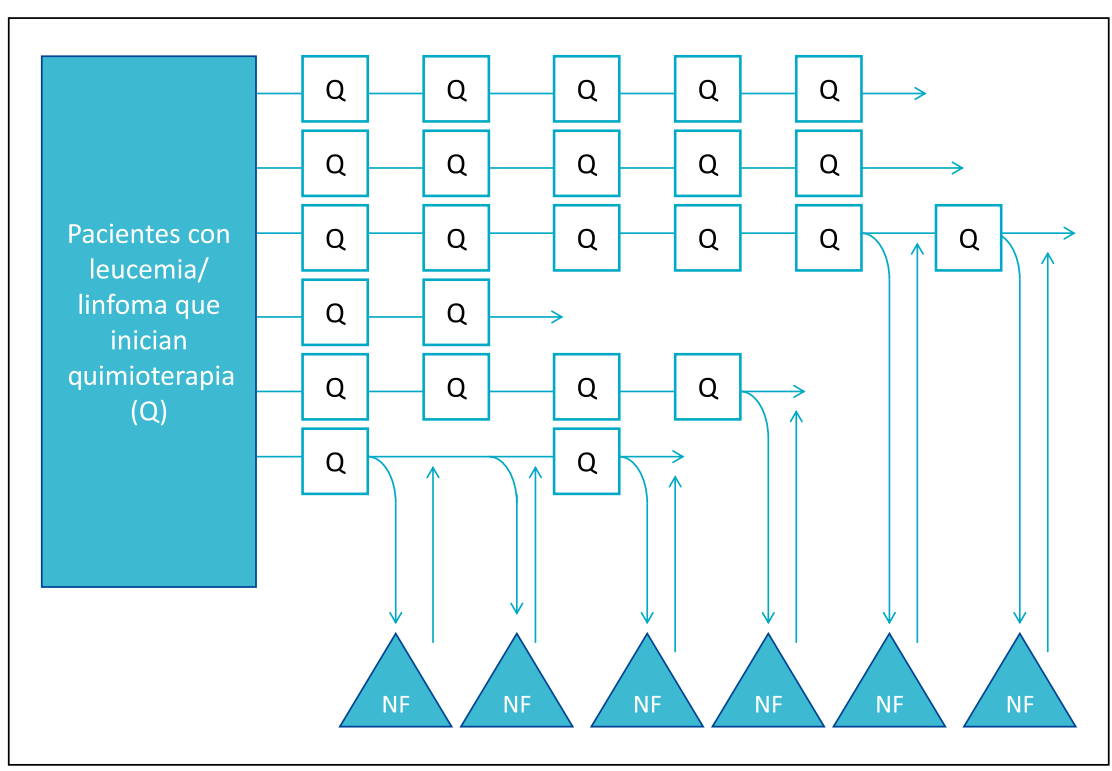

Figura 1. Representación esquemática del seguimiento de la cohorte de pacientes con LA o L identificados que fueron seguidos con posterioridad a cada ciclo de QMT (Q), registrando el desarrollo de NF, y luego continuando su seguimiento a través del tiempo.

Como objetivos secundarios se plantearon:

- Determinar los factores de riesgo para desarrollar NF.

- Determinar la frecuencia de IBI e IFI y factores asociados a su desarrollo.

- Reconocer factores asociados a mortalidad por episodios de NF.

Este artículo corresponde a la presentación y análisis de resultados del proyecto con financiamiento FONIS SA09I211.

\section{Pacientes y Métodos}

\section{Diseño}

Estudio prospectivo, de vigilancia de episodios de $\mathrm{NF}$ en una cohorte de pacientes adultos con LA o L diagnosticados y tratados en el Hospital Clínico Pontificia Universidad Católica de Chile (HCPUC) y Hospital Dr. Sótero del Río (HSR), hospitales privado y público respectivamente, de la ciudad de Santiago.

Se utilizó como definición de episodio de NF un recuento absoluto de neutrófilos $(\mathrm{RAN}) \leq 500$ céls $/ \mathrm{mm}^{3}$ y temperatura axilar $\geq 38^{\circ}$.

\section{Pacientes}

Se calculó un tamaño de muestra de 130 pacientes asumiendo un nivel de confianza de $95 \%$ y un error de estimación de $8,2 \%$ en la incidencia de NF. Se identificaron pacientes $\geq 18$ años con diagnóstico de leucemia mieloide aguda (LMA) o leucemia linfática aguda (LLA) o linfoma de Hodgkin (LH) o linfoma no Hodgkin (LNH), previo a recibir su primera QMT. Se excluyeron pacientes en recaída de $\mathrm{LA} / \mathrm{L}$ o previamente tratados, leucemias crónicas y episodios de NF durante trasplante de precusores hematopoyéticos. Se reclutaron pacientes desde abril de 2010 hasta noviembre de 2011 y el seguimiento de episodios de NF hasta enero de 2012.

El estudio fue aprobado por los comités de ética de ambos hospitales.

\section{Métodos}

Los pacientes fueron identificados en los servicios de hemato-oncología por vigilancia activa en cada centro (JF y PF) y reporte de los investigadores hematólogos (PB y $\mathrm{HR}$ ). Aquellos identificados fueron invitados a participar; quienes aceptaron ingresar, firmaron su consentimiento informado, dando inicio a la fase de recolección de datos demográficos, enfermedad oncológica, co-morbilidades e índice de Charlson ${ }^{14,15}$, Karnofsky ${ }^{16}$ y plan de QMT. Se mantuvieron en seguimiento con posterioridad a la administración de QMT. Los episodios de NF fueron detectados a través de una búsqueda activa de casos, realizándose una recopilación de variables clínicas, puntaje Apache $\mathrm{II}^{17}$, score de riesgo $\mathrm{MASCC}^{7}$, datos de laboratorio e imágenes y terapia, hasta la resolución del episodio continuando su seguimiento inter-ciclos de QMT hasta el cierre del estudio, alta o fallecimiento (Figura 1).

En base a los resultados de los exámenes clínicos y de laboratorio se clasificó a los episodios de NF en las siguientes categorías:

Infección microbiológicamente documentada (IMD) si se identificaba alguna especie bacteriana en el hemocultivo, cultivo corriente de cavidad estéril o muestra clínica apropiada de secreción, registrándose como episodio de IBI, viral (mediante técnicas de inmunofluorescencia, biología molecular o cultivo viral), o causa fúngica (hemocultivo positivo), cultivo de hongos o positividad del marcador de IFI galactomanano. Estas infecciones fúngicas, a su vez, fueron registradas como IFI posible, probable o probadas según las definiciones EORTC/MSG ${ }^{18}$.

Infección clínicamente documentada (ICD) si en la presentación clínica o radiológicamente se identificó un foco de infección. Se registró usando criterios de vigilancia de infecciones ${ }^{19}$.

Fiebre de origen desconocido (FOD) si no se identificó IMD ni ICD.

\section{Análisis estadístico}

Para determinar la incidencia de NF global y por grupos se utilizó la relación entre el número de episodios de NF como numerador y la sumatoria de número de días de observación posteriores a la administración de QMT, medidos entre el primer día de administración del ciclo y el día anterior de la siguiente administración, o el 
alta oncológica, fallecimiento o cierre del estudio como denominador.

Los resultados de las variables nominales se expresaron en porcentajes y de las numéricas, en mediana y rango. Para el cálculo de los intervalos de confianza se usó un nivel de 95\%. Para su análisis se utilizó el software SPSS versión 17.0 y el software de libre distribución R versión 2.8.1.

Para las comparaciones se aplicaron los test $\mathrm{t}$ de student, $\chi^{2}$ y test para comparar incidencias, considerándose como diferencia estadísticamente significativa el valor-p menor a 0,05. Cuando se utilizó test de hipótesis usando la misma muestra, se corrigió el nivel de la prueba (error tipo I) usando el método de $\operatorname{Sidak}^{20}$, en cuyo caso al usar la misma muestra para testear seis pruebas de hipótesis independientes se consideró como diferencia estadísticamente significativa el valor-p $<0,0085$ calculado por el método de Monte Carlo ${ }^{21}$. Se ajustaron modelos lineales generalizados para desenlace binario para establecer factores asociados a NF, IBI e IFI.

\section{Resultados}

Se identificaron 139 pacientes, 60 en el HCPUC y 79 en el HSR, pero se excluyeron 9 ( 1 del HCPUC y 8 del HSR) que rechazaron participar, por lo que finalmente se incluyeron datos de 130 pacientes que fueron seguidos en el tiempo. Sus características generales se detallan en la Tabla 1.

Co-morbilidades: Treinta y ocho $(29,2 \%)$ de los pacientes presentaron al menos una co-morbilidad, siendo las más frecuentes diabetes mellitus 14 (10,7\%), infección por VIH $9(6,9 \%)$ y cardiopatía coronaria $7(5,3 \%)$; por subgrupos, diabetes mellitus fue la co-morbilidad más frecuente en pacientes con LA y VIH en L. El índice de Charlson resultó 3 [2-7] en pacientes con LA vs 5 [3-9] en pacientes con $\mathrm{L}(\mathrm{p}<0,001)$.

Incidencia de NF: Los 130 pacientes recibieron 717 ciclos de QMT, sumando 16.247 días de seguimiento posteriores a su administración. Se identificaron 105 episodios de NF, resultando una incidencia de NF de 0,65 [IC 95\% (0,53-0,78)] por 100 días de observación post QMT. La incidencia fue significativamente mayor en los pacientes con LA vs aquellos con L: 1,31 [1,03-1,63] vs $0,25[0,17-0,37]$, (p: 0,001), en LMA vs LLA y en LNH vs LH (Tabla 2). La gradiente de mayor a menor incidencia de NF $(\mathrm{LMA}>\mathrm{LLA}>\mathrm{LNH}>\mathrm{LH})$ fue similar en ambos centros.

Los 105 episodios de NF se presentaron en 65 de los 130 pacientes incluidos, es decir, 50\% de los pacientes presentó al menos un episodio de NF, con mayor fre- cuencia en los pacientes con LA que L 47 (84\%) vs 18 $(24 \%),(p<0,0001)$.

Factores asociados al desarrollo de NF: Al comparar las características de los pacientes que presentaron $\mathrm{NF}$ con aquellos que no la presentaron, no se observaron diferencias en edad: (55 [19-85] vs 55 [16-81] años), sí una leve mayor frecuencia en el género masculino: 56,9 vs $50,9 \%$, menor presencia de co-morbilidades: 27,7 vs $32,3 \%$, menor índice de Karnofsky: 70 [50-90] vs 80 [50-90] y significativamente mayor frecuencia de LA como patología de base: 72,3 vs $15,3 \%$ ( $p<0,0001)$. En el análisis multivariado que incluyó edad, sexo masculino, número de ciclos y LA como patología oncológica de base, demostró asociación estadísticamente significativa de desarrollo de NF con el diagnóstico de LA.

\begin{tabular}{|lc|}
\hline \multicolumn{2}{|c|}{$\begin{array}{l}\text { Tabla 1. Características generales de } 130 \\
\text { LA o L incluidos en el estudiontes con }\end{array}$} \\
Edad (años) & $55[16-85]$ \\
Género masculino & $70(53,8)$ \\
Centro de atención HCPUC/HSR & $59(45) / 71(55)$ \\
Leucemia aguda & $56(43,1)$ \\
• Leucemia mieloide aguda & $37(28,5)$ \\
• Leucemia linfática aguda & $19(14,6)$ \\
Linfoma & $74(56,9)$ \\
• Linfoma no Hodgkin & $60(46,1)$ \\
• Linfoma de Hodgkin & $14(10,8)$ \\
Karnofsky al diagnóstico de LA/L & $70[50-90]$ \\
Charlson al diagnóstico de: & \\
• Leucemia aguda & $3[2-7]$ \\
• Linfoma & $5[3-9]$ \\
n total de ciclos de QMT observados & 717 \\
n de ciclos de QMT por paciente & $5,5[1-14]$ \\
\hline
\end{tabular}

Tabla 2. Incidencia de episodios de NF en los 100 días de seguimiento post QMT en leucemia aguda y linfoma

\begin{tabular}{|lcccc|}
\hline Patología & $\begin{array}{c}\text { Episodios de } \\
\text { NF (n) }\end{array}$ & $\begin{array}{c}\text { Días post } \\
\text { QMT }\end{array}$ & $\begin{array}{c}\text { Incidencia x } \\
\text { 100 días }\end{array}$ & [IC (95\%)] \\
Global & 105 & 16.247 & 0,65 & {$[0,53-0,78]$} \\
Leucemia aguda & 79 & 6.048 & 1,31 & {$[1,03-1,63]$} \\
- Leucemia linfática aguda & 21 & 2.887 & 0,73 & {$[0,45-1,11]$} \\
- Leucemia mieloide aguda & 58 & 3.161 & 1,83 & {$[1,39-2,37]$} \\
Linfoma & 26 & 10.199 & 0,25 & {$[0,17-0,37]$} \\
- Linfoma de Hodgkin & 2 & 2.653 & 0,08 & {$[0,01-0,27]$} \\
- Linfoma no Hodgkin & 24 & 7.546 & 0,32 & {$[0,20-0,47]$} \\
\hline
\end{tabular}


Tabla 3. Características generales de los 105 episodios de neutropenia febril (NF)

\begin{tabular}{|c|c|}
\hline & n (\%) Mediana [rango] \\
\hline Edad (años) & 56 [19-85] \\
\hline Género masculino & $64(60,9)$ \\
\hline Co-morbilidades ( $\geq 1$ ) & $35(33,3)$ \\
\hline Karnofsky al diagnóstico de NF & $70[50-90]$ \\
\hline Centro de atención HCPUC & $70(66,6)$ \\
\hline LA/L & $79(75,2) / 26(24,8)$ \\
\hline $\begin{array}{l}\text { Episodios de NF por paciente } \\
\text { - } n \text { de pacientes con } 1 \text { episodio } \\
\text { - } n \text { de pacientes con } 2 \text { episodios } \\
\text { - } n \text { de pacientes con } \geq 3 \text { episodios } \\
\text { Total de pacientes con episodios de NF }\end{array}$ & $\begin{array}{l}1[1-4] \\
37 \\
19 \\
9 \\
65\end{array}$ \\
\hline $\mathrm{n}$ ciclos de QMT previo al 1er episodio de NF & $2[1-13]$ \\
\hline Evaluación por infectólogo & $70(66,7)$ \\
\hline Hospitalización en $<24$ h de inicio de fiebre & $74(70,5)$ \\
\hline $\begin{array}{l}\text { Estado de LA a la presentación de NF ( } n=79) \\
\text { - Inducción } \\
\text { - Remisión } \\
\text { - Persistencia } \\
\text { - Recaída }\end{array}$ & $\begin{array}{rr}38 & (48,1) \\
35 & (43,7) \\
4 & (5,0) \\
2 & (2,5)\end{array}$ \\
\hline $\begin{array}{l}\text { Estado de } L \text { a la presentación de NF }(n=26) \\
\text { - Inducción } \\
\text { - Remisión } \\
\text { - Progresión } \\
\text { - Recaída }\end{array}$ & $\begin{array}{rr}14 & (53,8) \\
9 & (34,6) \\
1 & (3,6) \\
2 & (7,7)\end{array}$ \\
\hline Portación de CVC & $72(67,9)$ \\
\hline Mucositis & $32(30,2)$ \\
\hline $\begin{array}{l}\text { APACHE-II al diagnóstico de NF } \\
\text { - Leucemia aguda } \\
\text { - Linfoma }\end{array}$ & $\begin{array}{l}17[9-36] \\
19[10-29]\end{array}$ \\
\hline $\begin{array}{l}\text { APACHE-II al día } 3 \text { de NF } \\
\text { - Leucemia aguda } \\
\text { - Linfoma }\end{array}$ & $\begin{array}{l}16[7-36] \\
17[9-28]\end{array}$ \\
\hline
\end{tabular}

\section{Tabla 4. Etiología de los 105 episodios de NF en pacientes con LA y L}

\section{n $(\%)$}

Episodios con infección microbiológicamente documentada*\#

$46(43,8)$

Infección bacteriana invasora (bacteriemia)

- Bacteriemia diagnosticada del día 0 al 5

- Bacteriemia diagnosticada después del día 5

$33(31,4)$

27

6

Episodios con infección viral

$5(4,8)$

Infección fúngica invasora

- Infección fúngica diagnosticada del día 0 al 5

- Infección fúngica diagnosticada después del día 5

Episodios con Infección clínicamente documentada\#

$21(20)$

9

11

$34(32,4)$

Fiebre de origen desconocido

$38(36,2)$

*Incluye sumatoria de episodios con infección bacteriana, viral o fúngica. \#Incluye 13 episodios con infección clínicamente o microbiológicamente documentada simultánea.
En particular, entre los 47 pacientes con LA que desarrollaron NF vs 9 que no la presentaron, se observó discreta mayor edad: 50 [19-77] vs 47,5 [19-77] años, similitud en predominio del género masculino: $27(57,4 \%)$ vs $5(55,6 \%)$ y menor frecuencia de co-morbilidades: $11(23,4 \%)$ vs $4(44,4 \%),(p: 0,22)$. Por otra parte, entre 17 pacientes con L que presentaron NF vs 47 que no la desarrollaron, se observó mayor edad: 63,5 [20-85] años vs 57 [16-81] años, mayor frecuencia del género masculino: $9(52,9 \%)$ vs $22(46,8 \%)$ y mayor frecuencia de co-morbilidades: $6(35,2 \%)$ vs $14(29,8 \%)$ pero, sin diferencia estadísticamente significativa.

Características y estratificación de riesgo de los episodios: Las características generales de los episodios se presentan en la Tabla 3. El tiempo entre inicio de QMT y NF fue de 11 (1-40) días. En 31 (29,2\%) episodios existía el antecedente de al menos alguna otra NF y la mayor frecuencia de los episodios de NF se presentó durante la fase de inducción.

De acuerdo a la estratificación por MASCC, 44 $(41,9 \%)$ de los episodios fueron catalogados de alto riesgo (AR) vs $61(58,1 \%)$ de bajo riesgo (BR), con puntaje de 20 [13-19] vs 23 [21-26]. En los episodios de AR vs BR, los pacientes presentaron significativamente mayor edad: 62 [20-81] años vs 51 [19-85] años, (p: 0,04); género femenino más frecuente: $24(54,5 \%)$ vs $18(29,1 \%)$, (p: 0,01$)$; mayor frecuencia de hipotensión arterial: 25 $(56,8 \%)$ vs $8(12,9 \%),(\mathrm{p}<0,0001)$; mayor frecuencia de RAN < 100 céls/ $/ \mathrm{mm}^{3}: 40$ (90,9\%) vs 47 (75,8\%), (p: $0,007)$. Aunque sin diferencia estadísticamente significativa, el grupo de $\mathrm{AR}$ presentó fiebre y proteína $\mathrm{C}$ reactiva (PCR) más elevada: $38,4 \mathrm{mg} / \mathrm{dl}[36-40,5]^{\circ} \mathrm{C}$ vs 37,7 [36,5-39,9] ${ }^{\circ} \mathrm{C}$, (p: 0,3$)$; y $17,6[2,1-75]$ vs $10,3[0,7-64]$ (p: 0,1$)$, respectivamente. Al contrario no se observaron diferencias en co-morbilidades, LA como patología de base, mucositis, taquicardia, saturación de $\mathrm{O}_{2}<90 \%$, hematocrito, recuentos de plaquetas o glicemia.

En relación al puntaje Apache II al día 1 de NF fue de 19 [10-29] en L vs 17 [9-36] en LA, (p: 0,04). Los parámetros clínicos y de laboratorio del día 1 vs el día 3 de NF muestran disminución de la taquicardia: 67 vs $37 \%$ ( $<<0,0001)$ e hipotensión arterial: 31,1 vs $18,8 \%$ (p: 0,05$)$; menor intensidad de la fiebre: $38,3^{\circ} \mathrm{C}[36-$ $40,5]^{\circ} \mathrm{C}$ vs $38^{\circ} \mathrm{C}[36-40,1]^{\circ} \mathrm{C}$, (p: 0,3 ); aumento de la PCR: $11,8[0,76-75,4] \mathrm{mg} / \mathrm{dL}$ vs $20,5[1,44-98,6] \mathrm{mg} / \mathrm{dL}$, (p: 0,02$)$ y disminución en el score Apache II: $17( \pm 4,6)$ vs $16( \pm 4,3),(p=0,04)$. En cambio, no se observaron variaciones en hematocrito, recuento de linfocitos, glicemia, nitrógeno ureico o creatininemia.

Etiología: En 67 (63,8\%) episodios se documentó infección microbiológica y/o clínicamente y en 38 (36,2\%) episodios correspondió a FOD (Tabla 4). 
En 34 episodios con ICD se identificaron 45 focos de infección, es decir, en algunos episodios se constató más de un foco clínico. Los más frecuentes fueron foco gastrointestinal $16(35,5 \%)$, respiratorio $13(28,9 \%)$, piel $4(8,9 \%)$ y otros menos frecuentes como focos osteoarticular, hepato-biliar y urinario.

Infecciones bacterianas y fúngicas invasoras. Factores asociados: Hubo 33 (31,4\%) IBI con hemocultivos positivos, observados mayoritariamente durante los primeros cinco días de NF. No hubo identificación bacteriana en muestras de líquidos estériles. Entre las bacterias identificadas en hemocultivos predominaron la cocáceas grampositivas por sobre los bacilos gramnegativos (Tabla 5). La IBI fue más frecuente en LA que en L: $28(35,4 \%)$ vs $5(19,2 \%)$ (p: 0,09), con mayor número de bacteriemias en LMA $23(39,7 \%)$ vs LLA $5(23,8 \%)$ (p: 0,2$)$ y en LNH: $5(20,8 \%)$ vs $0(0 \%)$ en LH, (p: 1$)$.

En 21 (20\%) episodios se diagnosticó IFI con discreta mayor frecuencia después del quinto día. Predominó aspergilosis $17(80,9 \%)$ sobre candidiasis $3(14,2 \%)$ y

\begin{tabular}{|c|c|}
\hline & n $(\%)$ \\
\hline Cocaceas grampositivas & $23(60,5)$ \\
\hline Staphylococcus coagulasa negativa & $11(28,9)$ \\
\hline Streptococcus sp. & $5(13,2)$ \\
\hline Staphylococcus aureus & $3(7,9)$ \\
\hline Enterococcus faecium & $3(7,9)$ \\
\hline Corynebacterium sp. & $1 \quad(2,6)$ \\
\hline Bacilos gramnegativos & $15(39,5)$ \\
\hline Escherichia coli & $8(21)$ \\
\hline Klebsiella pneumoniae & $2(5,2)$ \\
\hline Enterobacter cloacae & $2(5,2)$ \\
\hline Pseudomonas aeruginosa & $1(2,6)$ \\
\hline Citrobacter freundii & $1(2,6)$ \\
\hline Acinetobacter baumannii & $1(2,6)$ \\
\hline
\end{tabular}

Tabla 6. Condición al cierre del estudio de 65 pacientes que presentaron episodios de NF

\begin{tabular}{|lc|}
\hline Condición & $\mathbf{n}(\mathbf{\%})$ \\
Vivo & $51(78,5)$ \\
- Con LA en remisión/L en remisión completa & $37(56,9)$ \\
- Con LA en persistencia/L remisión parcial & $13(20)$ \\
- Con LA recaída/L en progresión o recaída & $1(1,5)$ \\
Fallece & $14(21,5)$ \\
- Por infección & $4(6,2)$ \\
- Por progresión de enfermedad & $9(13,8)$ \\
- Por otra causa & $1(1,5)$ \\
\hline
\end{tabular}

1 (4,8\%) no identificado, calificándoseles como posible $10(47,6 \%)$, probable $8(38,1 \%)$ y probada $3(14,2 \%)$. La IFI fue más frecuente en LA que en L: 19 (25,3\%) vs $2(7,7 \%)(p: 0,06)$, con frecuencias similares en LMA y LLA (22 vs $23,8 \%$; p: 0,5$)$ y mayor en LNH vs LH (8,3\% vs $0 \%)(\mathrm{p}: 1)$.

IBI e IFI fueron más frecuentes, aunque sin diferencia estadísticamente significativa, en episodios de AR en comparación con BR 18 (40,9\%) vs $16(25,8 \%)$ (p: 0,1) y $11(25 \%)$ vs $10(16,4 \%)$ (p: 0,3), respectivamente.

Se ajustó un modelo lineal generalizado para la presencia o ausencia de IBI e IFI en función de un término constante, edad, sexo masculino, número de ciclos, temperatura al diagnóstico del episodio de NF, número de episodios de NF y puntaje MASCC.

Para IBI las variables número de ciclos y temperatura resultaron estadísticamente significativas, indicando que, a mayor número de ciclos, la probabilidad de IBI disminuía y que, a mayor temperatura, la probabilidad de IBI aumentaba. Si bien, el resto de las variables consideradas no resultaron estadísticamente significativas, se observó que a mayor edad, sexo femenino y mayor puntaje MASCC la probabilidad de IBI disminuía; en cambio, a mayor número de episodios, la probabilidad de IBI aumentaba.

Para IFI, la única variable estadísticamente significativa resultó ser el número de ciclos, indicando que a mayor número de ciclos la probabilidad de IFI disminuía. El resto de las variables no fueron estadísticamente significativas; sin embargo, se observó que a mayor edad, sexo masculino, mayor temperatura, mayor número de episodios de NF y mayor puntaje MASCC, la probabilidad de IFI disminuía.

Tratamiento, evolución, mortalidad por episodios de NF: En cada episodio se utilizaron dos [1-4] esquemas de antimicrobianos. Los más indicados inicialmente fueron ceftazidima o cefepima, asociados o no a aminoglucósidos, más vancomicina ( $33 \%$ en un primer esquema y $38 \%$ en un segundo esquema) o sin vancomicina $(25,5 \%$ en un primer esquema y $11,9 \%$ en un segundo esquema). En el tercer esquema predominó la indicación de carbapenémicos más vancomicina (60\%). En 19\% de los esquemas antimicrobianos se incluyó metronidazol.

En $45(42,9 \%)$ de los episodios fueron utilizados antifúngicos, siendo más frecuentemente indicados, voriconazol $18(40 \%)$ y fluconazol $15(33,3 \%)$, seguido por caspofungina $6(13,3 \%)$ y anfotericina-B desoxicolato y liposomal 2 (4,4\%), cada una. En 4 (8,8\%) se usó asociaciones de antifúngicos: voriconazol más anfotericina o caspofungina. En cuanto al motivo de su prescripción, fue empírica 18 (40\%) y precoz o presuntivo (del inglés "pre-emptive") en 18 (40\%), y con menor frecuencia como profilaxis primaria o secundaria en seis $(13,3 \%)$ de los 
episodios; sólo en tres casos $(6,7 \%)$ fue indicado como tratamiento dirigido de una IFI probada.

En $44(41,5 \%)$ de los episodios de NF se utilizó factores estimulantes de colonia. En 12 (11,3\%) episodios la evolución fue desfavorable requiriendo traslado a una unidad de mayor complejidad, con mayor frecuencia en los episodios de AR que BR por MASCC: 11 (25\%) vs 1 $(1,6 \%)$ (p: 0,0002), y a mayor APACHE II 18,6 $\pm 5,0$ vs $16,4 \pm 3,9,(p: 0,01)$. La mediana de fiebre fue 4 [1-32] días, de neutropenia 8 [1-30] días y de hospitalización 14 [3-83] días.

En el análisis multivariado de la condición de egreso de los episodios de NF por subgrupos de patología oncológica de base se identifican asociaciones estadísticamente significativas de LA con IFI, evolución desfavorable y AR y en caso de L, con días de neutropenia.

Al cierre del estudio la mortalidad global entre los 130 pacientes incluidos fue de $25(19,2 \%)$ pacientes, con mayor mortalidad los que presentaron NF vs los que no la presentaron, $14(21,5 \%)$ vs $11(16,9 \%)(p=0,3)$. Sin embargo, sólo en cuatro $(6,2 \%)$ fue directamente relacionado a infección (Tabla 6 ), IBI en un paciente $(25 \%)$ con sepsis por Escherichia coli e IFI en tres pacientes (75\%) correspondiendo a una candidiasis y dos aspergilosis invasora. La mortalidad fue mayor en los episodios de AR que los de BR, siete $(15,9 \%)$ vs cero $(0 \%)$, (p: 0,001$)$.

\section{Discusión}

El objetivo principal de este estudio fue logrado al determinar la incidencia de NF en pacientes adultos con LA/L en QMT; 0,65 [0,53-0,78] por 100 días de observación post QMT. La incidencia fue significativamente mayor en los pacientes con LA vs L: 1,31 [1,03-1,63] vs 0,25 [0,17-0,37], (p: 0,001), en LMA vs LLA $(1,83$ $[1,39-2,37]$ vs $0,73[0,45-1,11]$, (p: 0,0002$)$ y en LNH vs LH $(0,32[0,2-0,47]$ vs 0,08 [0,01-0,27], (p: 0,033). Esto se traduce en que $50 \%$ de los pacientes presentó al menos un episodio de NF, con significativa mayor frecuencia en LA que L, 84 vs 24\%. Y específicamente mayores en LMA, seguido de LLA, luego LNH y la menor en LH, $89,74,27$ y $14 \%$, respectivamente.

La incidencia de NF no había sido precisada en nuestro medio y, desde un punto de vista epidemiológico, ha sido muy importante que en este estudio se incluyeran datos de dos centros, uno privado y otro público. De acuerdo a los resultados, se debe plantear que en ambos centros la estrategia para disminuir episodios de NF, IBI e IFI debe ser distinta para LA que para L; esto debería ser válido para otros centros públicos y privados del país.

En vista que LA resultó ser la patología oncológica que más se asoció a NF, debería priorizarse la implementación de estrategias de prevención específicas en estos pacientes. Adicionalmente, las IBI e IFI fueron más frecuentes en los episodios de NF de los pacientes con LA vs L: $28(35,4 \%)$ vs $5(19,2 \%)$ y $19(25,3 \%)$ vs $2(7,7 \%)$, respectivamente; en consecuencia se hace necesario revisar las actuales estrategias de prevención de IBI e IFI en estos pacientes. Al contrario de un estudio anterior publicado por el HCPUC en el año $2009^{5}$, en la presente cohorte existe una mayor frecuencia de IBI por cocáceas grampositivas, si bien no fue posible identificar una causa específica para esta diferencia, Sí se debe hacer notar que está descrito en la literatura médica internacional el incremento de especies grampositivas sobre gramnegativas, hecho relacionado al uso de catéteres, mayor frecuencia e intensidad de mucositis debido a tipo de quimioterapias y uso de profilaxis antimicrobiana dirigida sobre bacilos gramnegativos ${ }^{22}$.

Dentro de la identificación de cocáceas grampositivas, se incluye la identificación de Staphylococcus aureus resistente a meticilina y Enterococcus faecium resistente a vancomicina, continuando el patrón nacional de incremento de estos agentes, lo cual obliga a mantener planes de vigilancia activa de las bacteriemias en estos pacientes en los diferentes centros a lo largo de Chile. Entre los bacilos gramnegativos, también se identificaron agentes resistentes como enterobacterias productoras de BLEE y Acinetobacter baumannii multiresistente, consistentes con reportes internacionales ${ }^{22}$, ante lo que vale el mismo comentario. Las bacteriemias por Pseudomonas spp mantienen una baja frecuencia; no obstante, su presencia ratifica la validez de iniciar empíricamente con esquemas antimicrobianos con actividad anti-pseudomonas. Si bien en algunos casos en el HSR se inicio tratamiento antibacteriano empírico, sin cobertura de Pseudomonas sp., se requiere datos muy consistentes de la ausencia de esta bacteria para prescindir de su cobertura en el esquema inicial.

Es necesario efectuar estudios para determinar factores de riesgo de bacteriemias por especies resistentes en pacientes hemato-oncológicos, que permitan diseñar algoritmos y esquemas antimicrobianos empíricos que aseguren su cobertura a la espera del informe de hemocultivos para ajustar esta terapia. Este punto cobra particular atención y, en ausencia de nuevos estudios, es importante mantener vigilancia epidemiológica de los episodios de NF de cada centro para plantear adaptaciones a las recomendaciones nacionales o internacionales de manejo anti-infeccioso para estos pacientes ${ }^{23}$. Estas adaptaciones no eximen de la necesidad de ser muy exigente en el cumplimiento de la normativa de control y prevención de infecciones asociadas a la atención en salud, para todo el equipo clínico que atiende a estos pacientes, haciendo particular hincapié en el cumplimiento de las precauciones estándares, las adicionales cuando corresponda y el adecuado uso de terapia antimicrobiana.

No se incluyó en el análisis el impacto de la profilaxis dado que su aplicación en cada centro es individualizada 
según sea el caso clínico y las preferencias del médico tratante. Para establecer conclusiones válidas se requiere un estudio que incluya un número necesario de pacientes expuestos para conocer los eventuales riesgos y beneficios. La validez de la profilaxis antibacteriana fue puesta de manifiesto en dos estudios clínicos publicados en el año 2005 que apoyan el uso de levofloxacina profiláctica en pacientes neutropénicos ${ }^{24,25}$; sin embargo, su aplicación fue cuestionada localmente luego de su publicación ${ }^{26}$, a la luz de las guías chilenas ${ }^{8}$. Más recientemente, las guías norteamericanas para el manejo de la NF recomiendan su uso en pacientes con mayor riesgo (en que se espera una neutropenia de $<100$ céls $/ \mathrm{mm}^{3}$ por más de 7 días) asociado a un programa de vigilancia de emergencia de resistencia a quinolonas ${ }^{24}$.

En otro aspecto, las IFI se presentaron en $20 \%$ de los episodios y se asociaron estadísticamente a la condición de egreso de los pacientes. Llama la atención la frecuencia de IFI diagnosticadas antes del quinto día de NF; esto podría obedecer al uso actual de las herramientas serológicas e imágenes que permiten diagnosticar precozmente estas infecciones. No se puede descartar, sin embargo, otros factores propios de los pacientes que determinan un mayor compromiso inmunológico como edad, co-morbilidades, así como también la quimioterapia empleada. Aspergilosis fue la infección fúngica más frecuente, de la misma manera que se reporta en la literatura científica nacional e internacional ${ }^{2,8,9,27}$, mientras que Candida spp., fue la segunda etiología. Solamente en un caso no se logró precisar una IFI específica; si bien el paciente presentaba imágenes sugerentes de infección por Aspergillus spp, el marcador galactomanana no apoyó el diagnóstico. Con estos datos, es necesario investigar el impacto de uso de profilaxis antifúngica activa sobre Aspergillus spp. En la actualidad, la mayor evidencia disponible es que el uso profiláctico de posaconazol durante la NF disminuye la incidencia de IFI y mortalidad de los pacientes ${ }^{28}$.

El modelo de estratificación de riesgos $\mathrm{MASCC}^{7}$ logra identificar dos grupos de pacientes, presentando mayor frecuencia de IBI, IFI, evolución desfavorable y mortalidad los episodios con AR por sobre los de BR. Su aplicación, sin embargo, no es tan simple ni totalmente objetiva. Originalmente este modelo fue diseñado para identificar pacientes con bajo riesgo de complicaciones, con miras a implementar su tratamiento ambulatorio. Nuestro estudio no fue diseñado para validar este proceder, los pacientes de AR y BR fueron manejados de manera similar; no obstante, en ausencia de una mejor herramienta discriminatoria, su uso puede ayudar a sugerir riesgos e intervenciones. Sería útil desarrollar un sistema que permita estratificar de manera más objetiva los pacientes con $\mathrm{NF}$, lo que requeriría un estudio con un diseño específico.
Los pacientes que cursan episodios de NF presentan discreta mayor mortalidad que aquellos que no la desarrollan; según GH Lyman y cols., la mortalidad fue al menos $15 \%$ superior en aquellos con NF vs aquellos que no la presentan ${ }^{29}$. Se podría plantear que la enfermedad más avanzada favorece el desarrollo de neutropenias más profundas y prolongadas dando origen a $\mathrm{NF}$ o, que el atraso de nuevos ciclos de QMT incide en la mayor progresión de la enfermedad de base disminuyendo la efectividad del tratamiento del cáncer, aunque no se puede descartar efectos sobre la progresión del cáncer producto de las infecciones invasoras y fenómenos inflamatorios asociados.

En resumen, nuestro trabajo proporciona los resultados de una cohorte nacional de LA y L que inician quimioterapia, permitiendo calcular la incidencia de NF en general y separadas por LA y L, determinar factores asociados a su desarrollo y la frecuencia de IBI e IFI durante el episodio de neutropenia. Es necesario evaluar el impacto de intervenciones destinadas a disminuir la incidencia de $\mathrm{NF}$, IBI e IFI, entre las que se debe incluir el beneficio y riesgo del uso sistemático de profilaxis antibacteriana y antifúngica en los subgrupos con mayor incidencia de episodios de NF.

\section{Resumen}

Introducción: La neutropenia febril (NF) es una complicación frecuente de pacientes sometidos a quimioterapia (QMT). Su presentación clínica es amplia, desde cuadros leves a sepsis grave con infección bacteriana invasora (IBI) o infección fúngica invasora (IFI), con gran impacto en el pronóstico y mortalidad de los pacientes. Pacientes y Métodos: Estudio prospectivo de episodios de NF en cohorte de pacientes adultos con leucemia aguda (LA) o linfoma (L) diagnosticados y tratados en el Hospital Clínico Pontificia Universidad Católica de Chile y Hospital Dr. Sótero del Río en Santiago, desde abril de 2010 hasta enero de 2012. Resultados: Se reclutaron 130 pacientes que presentaron 105 episodios de NF, con incidencia de 0,65 por 100 días de observación, mayor en LA que en L ( 1,31 vs 0,25, p: $0,001)$, documentándose etiología o foco infeccioso en $67(63,8 \%)$ de los episodios, con $33(31,4 \%)$ IBI y 21 (20\%) IFI. Hubo mortalidad relacionada a infección en 4 $(6,2 \%)$ pacientes. Conclusiones: Se define la incidencia de NF $(\mathrm{LA}>\mathrm{L})$ y frecuencia de IBI e IFI durante el episodio (LA $>$ L). Es necesario evaluar el impacto de intervenciones destinadas a disminuir la incidencia de $\mathrm{NF}$, entre las que se debe incluir el beneficio y riesgo del uso sistemático de profilaxis antibacteriana y antifúngica en los subgrupos de mayor riesgo. 


\section{Referencias bibliográficas}

1.- Bow E J. Infection in neutropenic patients with cancer. Crit Care Clin 2013; 29: 411-41.

2.- Menichetti F. Infectious complications in neutropenic cancer patients. Intern Emerg Med 2010; 5 (Suppl 1): S21-S25.

3.- Crawford J, Dale D C, Lyman G H. Chemotherapy-induced neutropenia: risks, consequences, and new directions for its management. Cancer 2004; 100: 228-37.

4.- Pettengell R, Shwenkglenks M, Leonard R, Bosly A, Paridaens R, Constenla M, et al. Neutropenia occurrence and predictors of reduced chemotherapy delivery: results from the INC-EU prospective observational European neutropenia study. Support Care Cancer 2008; 16: 1299-309.

5.- Rabagliati R, Fuentes G, Orellana E, Oporto J, Domínguez I, Benítez R, et al. Etiología de episodios de neutropenia febril en pacientes adultos con cánceres hematológicos y de órganos sólidos en el Hospital Clínico Universidad Católica, Santiago-Chile. Rev Chilena Infectol 2009; 26: 109-13.

6.- Intragumtornchai T, Sutheesophon J, Sutcharitchan P, Swasdikul D. A predictive model for life-threatening neutropenia and febrile neutropenia after the first course of CHOP chemotherapy in patients with aggressive non-Hodgkin's lymphoma. Leuk Lymphoma 2000; 37: 351-60.

7.- Uys A, Rapoport B L, Anderson R. Febrile neutropenia: a prospective study to validate the Multinational Association of Supportive Care of Cancer (MASCC) risk-index score. Support Care Cancer 2004; 12: 555-60.

8.- Santolaya M E, Rabagliati R, Bidart T, Paya E, Guzmán A M, Morales R, et al Consenso Manejo racional del paciente con cáncer, neutropenia y fiebre. Rev Chilena Infect 2005; 22 (Supl 2): S79-S113.

9.- Santolaya M E, Álvarez A M, Avilés C L, Becker A, Cofré J, Cumsille M A, et al. Early hospital discharge followed by outpatient management versus continued hospitalization of children with cancer, fever and neutropenia at low risk for invasive bacterial infection. J Clin Oncol 2004; 22: 3784-9.

10.- Santolaya M E, Álvarez A M, Avilés C L, Becker A, King A, Mosso C, et al. Predictors of severe sepsis, not clinically apparent during the first twenty-four hours of hospitalization in children with cancer, neutropenia and fever: a prospective, multicentes trial. Pediatr Infect Dis J 2008; 27: 538-43.

11.- Talcott J, Siegel R, Finberg R, Goldman L. Risk assessment in cancer patients with fever and neutropenia. A prospective, two-validation of a prediction rule. J Clin Oncol 1992; 10: 316-22.

12.- Puga B, Puga I, Cabrera M E, Undurraga M S, Guerra C, Urrejola G, Toro P. High risk febrile neutropenia in acute leukemia. The experience of a public hospital. National Program of Antineoplastic Drugs in Adult, Hospital del Salvador, 1991-2001. Rev Med Chile 2003; 131: 1023-30.

13.- Puga B, Molina J, Andrade A, Guerra C, Ardila A, Álvarez G. First ten hematopoietic stem cell transplants performed in the adult public health service in Chile. Rev Med Chile 2012; 140: 1207-12.

14.- Charlson M E, Pompei P, Ales K L, MacKenzie C R. A new method of classifying prognostic comorbidity in longitudinal studies: development and validation. J Chronic Dis 1987; 40: 373-83.

15.- Charlson M E, Charlson R E, Paterson J C, Marinopoulos S S, Briggs W M, Hollenberg J P. The Charlson comorbidity index is adapted to predict costs of chronic disease in primare care patients. J Clin Epidemiol 2008; 61: 1234-40.

16.- Abernethy A P, Wheeler J L, Currow D C. Utility and use of palliative care screening tools in routine oncology practice. Cancer J 2010; 16 : 444-60.

17.- Breslow M J, Badawi O. Severity scoring in the critically ill: Part 1-interpretation and accuracy of outcome prediction scoring systems. Chest 2012; 141: 245-52.

18.- De Pauw B, Walsh T J, Donnelly P, Stevens D A, Edwards J E, Calandra T, et al. Revised definitions of invasive fungal disease from the European Organization for Research and Treatment of Cancer/Invasive Fungal Infections Cooperative Group and the National Institute of Allergy and Infectious Diseases Mycoses Study Group (EORTC/MSG) Consensus Group. Clin Infect Dis 2008; 46: 1813-21.

19.- Horan T C, Andrus M, Dudeck M A. CDC/ NHSN surveillance definition of health careassociated infection and criteria for specific types of infections in the acute care setting. Am J Infect Control 2008; 36: 309-32.

20.- Sidak Z. Rectangular confidence regions for the means of multivariate normal distributions. J Am Statistical Ass, 1967; 62 (31): 626-33.

21.- Hope A C A. A simplified Monte Carlo significance test procedure. J Royal Statistical Soc. Series B (Methodological) 1968; 30 (3): 582-98.

22.- Montassier E, Batard E, Gastinne T, Potel $\mathrm{G}$, de La Cochetière M F. Recent changes in bacteremia in patients with cancer: a systematic review of epidemiology and antibiotic resistance. Eur J Clin Microbiol Infect Dis 2013 ; 32: 841-50.

23.- Freifeld A G, Bow E J, Sepkowitz K A, Boeckh M J, Ito J I, Mullen C A, et al. Clinical practice guideline for the use of antimicrobial agents in neutropenic patients with cancer: 2010 Update by the Infectious Diseases Society of America. Clin Infect Dis 2011; 52: e56-e93.

24.- Bucaneve G, Micozzi A, Menichetti F, Martino P, Dionisi M S, Martinelli G, et al. Levofloxacin to prevente bacterial infection in patients with cancer and neutropenia. N Engl J Med 2005; 353: 977-87.

25.- Cullen M, Steven N, Billingham L, Gaunt C, Hastings M, Simmonds P, et al. Antibacterial prophylaxis after chemotherapy for solid tumors and lymphomas. N Engl J Med 2005; 353: 98898.

26.- Rabagliati R, Bidart T, Santolaya M E. Uso de profilaxis antibiótica en pacientes neutropénicos. Rev Med Chile 2006; 134: 801-2.

27.- Rabagliati R R, Fuentes L G, Guzmán D A M, Orellana E U, Oporto J C, Aedo I, et al. Enfermedad fúngica invasora en pacientes hemato-oncológicos y receptores de trasplante de precursores hematopoyéticos bajo la perspectiva de los criterios diagnósticos EORTC/MSG. Rev Chilena Infectol 2009; 26: 212-9.

28.- Cornely O A, Maertens J, Winston D J, Perfect J, Ullmann A J, Walsh T J, et al. Posaconazole vs fluconazole or itraconazole prophylaxis in patients with neutropenia. N Engl J Med 2007; 356: 348-59.

29.- Lyman G H, Michels S L, Reynolds M W, Barron R, Tomic K S, Yu J. Risk of mortality in patients with cancer who experience febrile neutropenia. Cancer 2010; 116: 5555-63. 